\title{
FUENTES DE INFORMACIÓN QUE UTILIZAN LAS EMPRESAS PARA EL DESARROLLO DE ACTIVIDADES DE INNOVACIÓN EN ECUADOR
}

\author{
SOURCES OF INFORMATION USED BY COMPANIES FOR THE \\ DEVELOPMENT OF INNOVATION ACTIVITIES IN ECUADOR
}

\author{
Raúl Carpio Freire, MBA \\ Universidad Espíritu Santo - Ecuador \\ rcarpiof@uees.edu.ec \\ Susan Flores Armijos \\ Universidad Espíritu Santo - Ecuador
}

\section{RESUMEN}

Este artículo muestra las diferentes fuentes que las empresas usan para la innovación, analizando cada variable que se presenta mediante un estudio explicativo utilizando el método cualitativo. Sabiendo que la innovación es primordial para una empresa, se busca encontrar cual es la fuente o las fuentes de información que permite que se realice algo nuevo que hace que dicha empresa se mantenga pionera en el mercado.

Palabras claves: innovación, Ecuador, fuentes de información, desarrollo de productos.

\begin{abstract}
This article shows the different sources of information that companies in Ecuador use for innovation by analyzing each variable that occurs through an explanatory study using the quantitative method. Knowing that innovation is critical to a company, the aim is to find the source or sources of information that make it possible to do something innovative in order to keep the company's pioneering position in the market.
\end{abstract}

Keywords: innovation, prospective, source of information, market leadership.

Recibido: Febrero de 2015 Aprobado: Mayo de 2015

\section{Introducción}

La innovación es un tema importante para una empresa debido a que evita el estancamiento productivo y logra una expansión colectiva, ya que mediante la creación de nuevos productos nunca vistos en un mercado o la elaboración de nuevas estrategias para el manejo de las empresas estas puede mejorar notablemente sus actividades, acaparando mercados y creando ventajas competitivas.(Rivero, 2010).

Por lo general las personas dicen que innovar es tan solo tener una "gran idea", pero es más que eso. El proceso de innovación demanda una investigación previa, debe contar con fuentes reales, utilizando tecnología apropiada de la mano de teorías científicas, con la finalidad de que los resultados sean óptimos.(Salazar, 2013).

En 1995, la Senacyt, organización que actualmente se llama Senescyt, desarrolló el primer programa de ciencia y tecnología, dando como resultado 35 proyectos de investigación y desarrollo, 5 proyectos de servicios tecnológicos, 7 proyectos de inversión en infraestructura científica y tecnológica, 6 proyectos de modernización tecnológica del sector privado, para el fortalecimiento del sistema nacional. (INEC, 2013).

Según datos corroborados por la coordinación de planificación de la Senacyt, el gobierno central del Ecuador asignó $0.23 \%$ del producto interno bruto a temas relacionados con la innovación, más que los cuatro años precedentes; el 22\% fueron asignados a información básica, $69 \%$ a investigación aplicada y $9 \%$ investigación experimentada. (Dirección de Investigaciones y proyectos académicos-DIPA, 2012). Sin embargo, en el 2009, la Senacyt dio a conocer un debilitado sistema, con poca inversión 
en ciencia y tecnología, baja competencia y poca producción.

Según el INEC (Instituto Nacional de Estadísticas y Censo), clasifica la innovación como la incorporación de una empresa al mercado nacional o al mundo, un nuevo o mejorado bien o servicio, por último, todo cambio organizacional o método de comercialización.

La Innovación y Desarrollo (I $\neg$ +D) interno, es un trabajo sistemático creativo, tiene como objetivo desarrollar un nuevo conocimiento, ya sea científico o técnico, aplicando un conocimiento ya existente o desarrollado por otro. Se distinguen tres grandes categorías de investigaciones: la básica, que es generar un conocimiento abstracto o teórico dentro de un área científica o técnica; la aplicada, que es generar un nuevo conocimiento teniendo desde un principio la finalidad a la que se desea llegar y ; el desarrollo experimental, que es la fabricación y puesta a prueba de un prototipo. Por el contrario, la Innovación y Desarrollo $(I \neg \neg+D)$ externo, es el trabajo creativo que no se realiza dentro de la empresa o con el personal de la empresa, sino que es destinado a un tercero (Dirección de Investigaciones y proyectos académicos-DIPA, 2012).

Con estos antecedentes, el objetivo del presente artículo es revisar las principales fuentes de información que utilizan los gerentes y directivos en las empresas ecuatorianas para determinar las más recurrentes, como fuente de datos que permitan innovar, en algún eslabón de la cadena de producción o servicio.

\section{Metodología}

Mediante un estudio explicativo y cualitativo, se utilizó información que se extrajo de una base de datos del sitio web del Instituto Ecuatoriano de Estadísticas y Censos.(Secretaria de Educación Superior, Ciencia y tecnología, 2009-2011). Los datos se ingresaron en una hoja de Excel donde se escogió solo las variables a utilizar, las mismas que fueron mencionadas en el marco teórico, correspondientes a un periodo del 2009 al 2011.

\section{Resultados}

Un generador de fuentes de innovación es la observación de las actividades que se realizan dentro de la empresa. Estas observaciones se clasifican en: las actividades dentro de la empresa y las actividades que suceden fuera de ella. Se considera que las observaciones dentro de la empresa son mejores entendidas por los gerentes de las empresas o los encargados de realizar las inno- vaciones de la misma(Corma, 2013).

Dentro de la empresa, existen tres panoramas que se pueden tomar en cuenta al momento de inspirarse para innovar. El primero, es cuando una empresa tiene éxito o fracaso, de una manera inesperada. En este caso, esto les puede servir de inspiración para mejorar otros productos de la empresa, o en su defecto sabrán qué cosas no hacer $\mathrm{y}$ teniendo una pauta para mejorar o innovar.

El segundo panorama, es cuando existen discrepancias entre lo que la empresa está realizando y lo que de verdad se exige en el mercado. Es decir, al tener un contraste o discrepancia, los encargados tienen una razón sólida para poder innovar y solucionar sus inconvenientes(Alvarez, 2011).

El tercero y último panorama en la parte interina de la empresa enfocada en las fuentes de innovación, es casi obligatoria y de las más notadas. Se trata de la innovación por la necesidad. Esta fuente es muy observada, surge de la necesidad de mejorar algo debido a que un proceso interno no está funcionando como se debe (Herrera, 2013).

Se puede notar que las fuentes de inspiración para la innovación en una empresa, surgen a partir de un problema. Un obstáculo principal es el contenido o la solución de la inspiración(Gomez, 2011).

Según (Heinz, 2011)las fuentes de innovación que se usan más a menudo son:

- Preguntas al Cliente: Todos los días que estés contestando las preguntas de los clientes actuales y potenciales, a través de correo electrónico, teléfono, en persona, y en sus redes sociales. Cada vez que conteste una pregunta, es una entrada de innovación.

- Lecturas: No importa lo que estás leyendo. Libros, blogs, revistas, incluso los anuncios.

- Ideas distintas de otras personas: Cuando uno escucha ideas de otras personas y uno no está de acuerdo, es una gran oportunidad para analizarlas y lograr innovar a partir de ellas.

- Prensa: El simple de hecho de leer un libro o revista, o escuchar un reportaje, nuevas ideas dependiendo de las necesidades de las personas pueden aparecer.

- Conferencias, paneles y seminarios en línea: A partir de una sala de exposiciones, las ventas y técnicas de marketing de otros expositores que utilizan para tratar y las nuevas personas 
que se encuentran en la misma conferencia pueden ser fuentes de innovación.

- Twitter:Al tomar una palabra clave relevante para usted, su empresa o sus clientes, ponga un hashtag en frente de ella, y hacer una búsqueda en search.twitter.com. Vea de lo que otras personas están hablando en este momento. Valiosa fuente de innovación.

- Linked In Answers: Encuentra los temas y sub-temas más relevantes para su negocio y clientes, e inscribirse en los resúmenes por correo electrónico diario. Es como tener una lista completa de posibles fuentes de innovación en su bandeja de entrada todos los días.

- Las noticias: Simplemente escuchar las noticias puede traer grandes fuentes de inspiración para lograr de esa manera innovar de una mejor manera.

Las empresas, según datos del INEC en el periodo de 2009 a 2011, optaron por adquirir máquinas y equipos. Esto conlleva a la capacitación de sus empleados revitalizando la economía de gran escala y reduciendo costos.

Tabla 1. Porcentaje de empresas que realizaron actividades de innovación de producto y proceso durante el período de 2009 a 2011

\begin{tabular}{|l|l|}
\hline ACTIVIDADES DE INNOVACIÓN & \multicolumn{1}{l}{$\%$ EMPRESAS } \\
\hline Tecnología Desincorporada & $3,22 \%$ \\
\hline Ingeniería y Diseño Industrial & $3,82 \%$ \\
\hline I+D Externa & $4,56 \%$ \\
\hline Estudios de mercado & $6,39 \%$ \\
\hline Consultoría y Asistencia Técnica & $11,20 \%$ \\
\hline Hardware & $14,31 \%$ \\
\hline Software & $14,36 \%$ \\
\hline I+D Interna & $15,03 \%$ \\
\hline Capacitación & $20,18 \%$ \\
\hline $\begin{array}{l}\text { Adquisición de Maquinaria y equi- } \\
\text { pos }\end{array}$ & $31,22 \%$ \\
\hline
\end{tabular}

FUENTE: ENCUESTA DE ACTIVIDADES DE INNOVACIÓN

Como fuentes de información interna para el desarrollo de sus actividades de innovación, las empresas decidieron abarcar el área de administración y finanzas, con un porcentaje considerable del $72.58 \%$ y con menor porcentaje del $14.62 \%$ buscan información en empresas competidoras o diferentes empresas del mismo sector, dando como resultado que las empresas están creando sus propias investigaciones para resolver las deficiencias que tiene el mercado y generando soluciones propias en su demanda.
Tabla 2. Porcentaje de empresas que utilizaron fuentes de información interna para el desarrollo de sus actividades de innovación durante el período de 2009 a 2011

\begin{tabular}{|c|c|}
\hline $\begin{array}{l}\text { FUENTES INTERNAS DE INFOR- } \\
\text { MACIÓN }\end{array}$ & $\%$ EMPRESAS \\
\hline $\begin{array}{l}\text { Otras empresas del grupo o casa } \\
\text { matriz }\end{array}$ & $14,62 \%$ \\
\hline $\begin{array}{l}\text { Departamento de Investigación y } \\
\text { Desarrollo }(I+D)\end{array}$ & $24,60 \%$ \\
\hline Áreas de distribución & $41,56 \%$ \\
\hline Áreas de marketing & $43,59 \%$ \\
\hline Áreas de sistemas (TIC's) & $45,21 \%$ \\
\hline Áreas de producción & $54,54 \%$ \\
\hline Áreas de administración y finanzas & $72,58 \%$ \\
\hline
\end{tabular}

FUENTE: ENCUESTA DE ACTIVIDADES DE INNOVACIÓN

Uno de los principales actores, sin lugar a dudas, son las propias empresas, los emprendedores, ya sean grandes o pequeños, que se arriesgan a darle un giro diferente al negocio para que sus productos siempre sean escogidos por el cliente. En el gráfico 3 se puede observar el alto porcentaje $85.59 \%$ que ocupa la empresa, como principal innovador.

Tabla 3. Actores de la innovación de producto durante el período de 2009 a 2011 (expresado en porcentaje)

\begin{tabular}{|l|l|}
\hline ACTORES & $\%$ \\
\hline La propia empresa & $85,59 \%$ \\
\hline $\begin{array}{l}\text { La empresa en cooperación con } \\
\text { otra empresa o institución }\end{array}$ & $7,40 \%$ \\
\hline $\begin{array}{l}\text { La empresa, adaptando o mo- } \\
\text { dificando los bienes o servicios } \\
\text { originalmente desarrollados por } \\
\text { otra empresa o institución }\end{array}$ & $7,57 \%$ \\
\hline Otra empresa o institución & $2,79 \%$ \\
\hline
\end{tabular}

FUENTE: ENCUESTA DE ACTIVIDADES DE INNOVACIÓN

Gráfico 1. Actores de la innovación de producto durante el período de 2009 a 2011

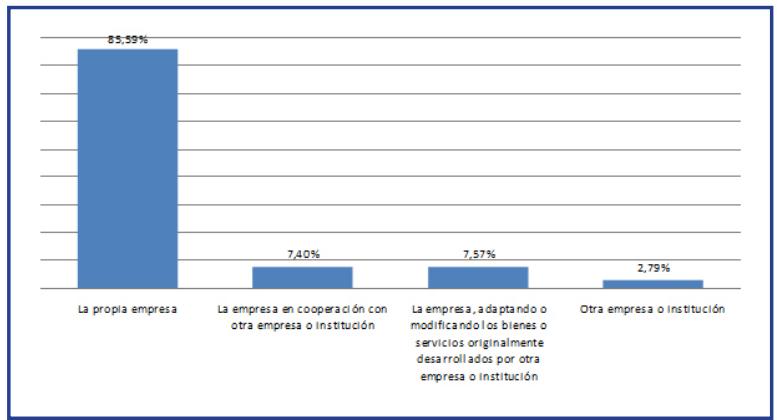

FUENTE: ENCUESTA DE ACTIVIDADES DE INNOVACIÓN

Como principal fuente de financiamiento, en el gráfico 4 , se puede observar casi del $100 \%$, que las empresas privadas, en un $66.68 \%$ utiliza su propio recurso para el desarrollo de las actividades de innovación y solo el $7.18 \%$ se ayuda con 
departamentos gubernamentales. Se puede observar en la tabla 4 , que otra de las fuentes de financiamiento que usan las empresas, son recursos del exterior y bancos privados.

Tabla 4. Fuentes de financiamiento para el desarrollo de actividades de innovación de producto y proceso durante el período de 2009 a 2011

\begin{tabular}{|l|l|}
$\begin{array}{l}\text { FUENTES DE } \\
\text { FINANCIAMIENTO }\end{array}$ & \multicolumn{1}{l}{$\begin{array}{l}\text { FINANCIAMIENTO } \\
(\%)\end{array}$} \\
\hline Apoyos Gubernamentales & $7,18 \%$ \\
\hline Banca privada & $16,68 \%$ \\
\hline $\begin{array}{l}\text { Recursos provenientes del } \\
\text { exterior }\end{array}$ & $8,01 \%$ \\
\hline Recursos propios & $66,96 \%$ \\
\hline Otras Fuentes & $1,16 \%$ \\
\hline
\end{tabular}

FUENTE: ENCUESTA DE ACTIVIDADES DE INNOVACIÓN

Gráfico 2. Fuentes de financiamiento para el desarrollo de actividades de innovación de producto y proceso durante el período de 2009 a 2011

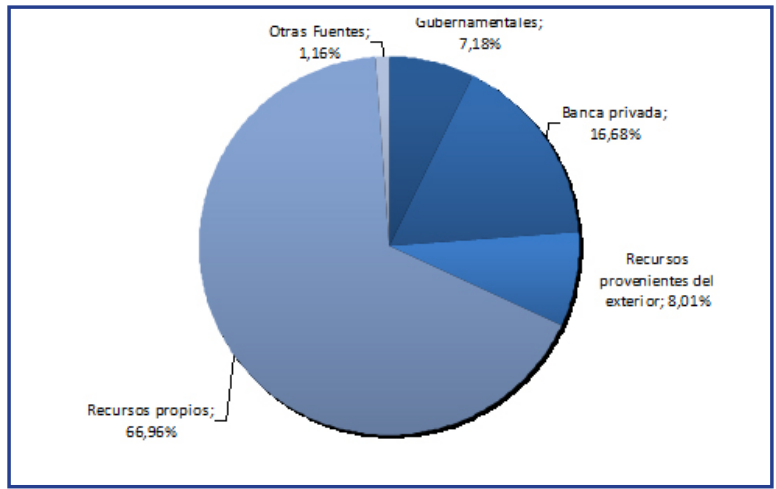

FUENTE: ENCUESTA DE ACTIVIDADES DE INNOVACIÓN

La determinante que hace que las empresas se arriesguen, motiven e interesen a las empresas a tomar la decisión de la innovación, es que de la demanda total del mercado el $52.91 \%$ de la población se siente insatisfecha por los productos actuales, el empresario toma esta variable como una oportunidad para llegar a los clientes, haciendo una mejora a su servicio o producto, mejorando también la calidad del producto ecuatoriano.

Tabla 5. Determinantes que motivaron la puesta en práctica de actividades de innovación de producto y proceso durante el período de 2009 a 2011

\begin{tabular}{|l|l|}
\hline DETERMINANTES & $\%$ \\
\hline Cambios en normas de propiedad intelectual & $5,12 \%$ \\
\hline Pautas regulatorias & $14,23 \%$ \\
\hline Procesos de certificación & $16,44 \%$ \\
\hline Problema técnico & $16,47 \%$ \\
\hline $\begin{array}{l}\text { Aprovechamiento de una idea o de novedades } \\
\text { cientificas y técnicas }\end{array}$ & $35,81 \%$ \\
\hline Amenaza de la competencia & $45,75 \%$ \\
\hline $\begin{array}{l}\text { Aprovechamiento de una idea generada al inte- } \\
\text { rior de la firma }\end{array}$ & $46,69 \%$ \\
\hline $\begin{array}{l}\text { Detección de una demanda total o parcialmente } \\
\text { insatisfecha en el mercado }\end{array}$ & $52,91 \%$ \\
\hline
\end{tabular}

FUENTE: ENCUESTA DE ACTIVIDADES DE INNOVACIÓN
Gráfico 3. Determinantes que motivaron la puesta en práctica de actividades de innovación de producto y proceso durante el período de 2009 a 2011

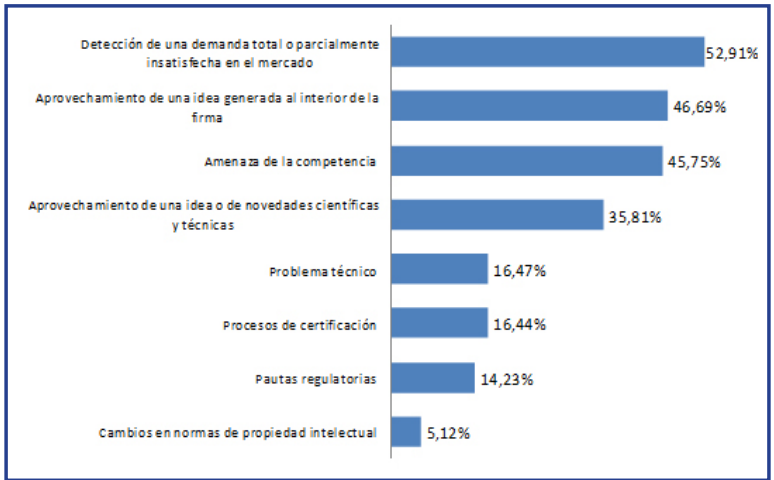

FUENTE: ENCUESTA DE ACTIVIDADES DE INNOVACIÓN

El aumento en la capacidad de producir nuevos productos o sus mejoras incrementó en un $78.39 \%$, siendo las variables antes mencionadas factores que complementaron que las actividades a desarrollar, aumenten parcialmente. Al adquirir la empresa su propia maquinaria y ser su inversionista principal, hace que la economía aumente generando campos de trabajo simultáneos al crecimiento de la producción.

Tabla 6. Impacto de las innovaciones introducidas, durante el período de 2009 a 2011

\begin{tabular}{|l|l|}
\hline \multicolumn{2}{|l|}{ IMPACTO DE INNOVACIÓN } \\
\hline $\begin{array}{l}\text { Reducción de los costos de materiales y energía } \\
\text { por unidad de }\end{array}$ & $55,20 \%$ \\
\hline $\begin{array}{l}\text { Reducción de los costos de producción por } \\
\text { unidad de producción }\end{array}$ & $58,74 \%$ \\
\hline Reducción de los impactos ambientales & $59,92 \%$ \\
\hline $\begin{array}{l}\text { Mejora de la salud o seguridad ocupacional de } \\
\text { sus empleados }\end{array}$ & $71,44 \%$ \\
\hline Ingreso a nuevos mercados & $72,44 \%$ \\
\hline $\begin{array}{l}\text { Reemplazo de los productos o procesos desac- } \\
\text { tualizados }\end{array}$ & $74,43 \%$ \\
\hline $\begin{array}{l}\text { Mejora de la flexibilidad para producir bienes o } \\
\text { servicios }\end{array}$ & $77,51 \%$ \\
\hline $\begin{array}{l}\text { Aumento de la capacidad para producir bienes o } \\
\text { servicios }\end{array}$ & $78,39 \%$ \\
\hline Aumento de la variedad de bienes o servicios & $78,73 \%$ \\
\hline Incremento de la participación de mercado & $81,00 \%$ \\
\hline Mejora de la calidad de bienes o servicios & $89,49 \%$ \\
\hline
\end{tabular}

FUENTE: ENCUESTA DE ACTIVIDADES DE INNOVACIÓN

Gráfico 4. Impacto de las innovaciones introducidas, durante el período de 2009 a 2011

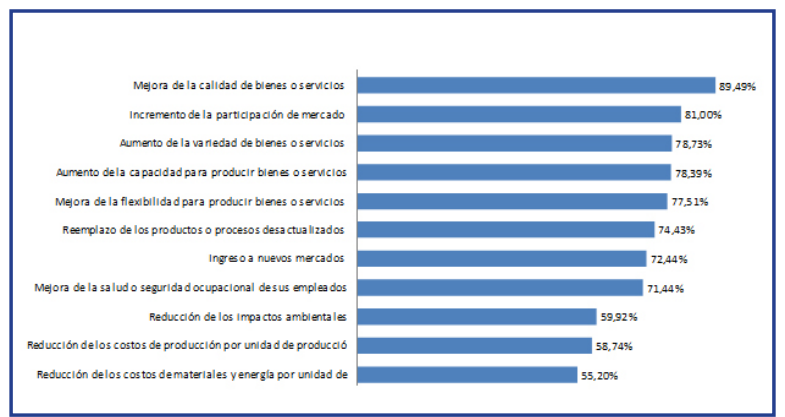

FUENTE: ENCUESTA DE ACTIVIDADES DE INNOVACIÓN 
EI INEC muestra un porcentaje del $58.88 \%$ en el mercado que posesiona a las empresas innovadoras en contra de posibles amenazas a las que se enfrentaría este sector, ocupando la mitad y un octavo de la proporción, se puede decir que en nuestra demanda las empresas están utilizando sus recursos al más alto nivel de ejecución, empleando nuevos planes estratégicos para su desarrollo.

Tabla 7. Porcentaje de empresas según clasificación (innovadoras, potenciales, no innovadoras), durante el período de 2009 a 2011

\begin{tabular}{|l|l|}
\hline CLASIFICACIÓN & $\%$ \\
\hline Empresas innovadoras & $58,88 \%$ \\
\hline Empresas potencialmente innovadoras & $1,94 \%$ \\
\hline Empresas no-innovadoras & $39,18 \%$ \\
\hline
\end{tabular}

FUENTE: ENCUESTA DE ACTIVIDADES DE INNOVACIÓN

Gráfico 5. Porcentaje de empresas según clasificación (innovadoras, potenciales, no innovadoras), durante el período de 2009 a 2011

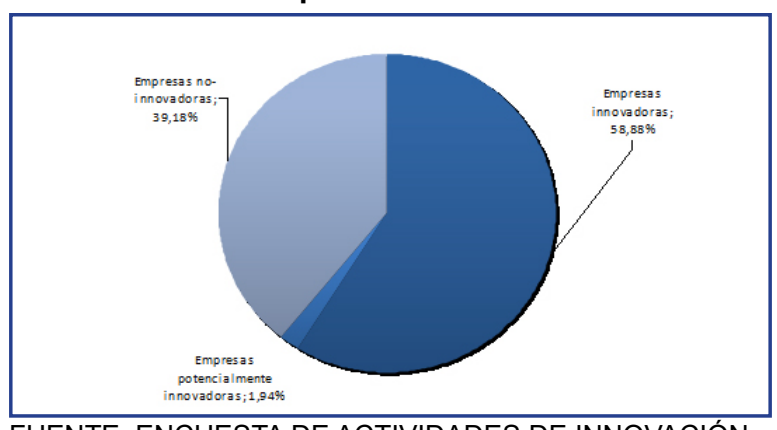

FUENTE: ENCUESTA DE ACTIVIDADES DE INNOVACIÓN

Al gasto que conlleva la implementación de ser innovador en el país, el INEC lo ha clasificado de dos maneras; una que abarca la investigación y desarrollo, es decir el I+D con un valor de $\$ 280$ millones, siendo el 2010 el año en que más se invirtió en el sector, por otro lado están las actividades de ciencia y tecnología (ACT) con un valor mayor de $\$ 316$ millones en el mismo año.

Tabla 8. Gasto en ciencia y tecnología ( Valores en millones de dólares corrientes)

\begin{tabular}{|l|l|l|l|}
\hline & 2009 & 2010 & 2011 \\
\hline I+D (Investigación y Desarrollo) & 246,7 & 280,3 & 269,5 \\
\hline $\begin{array}{l}\text { ACT (Actividades de Ciencia y } \\
\text { Tecnología) }\end{array}$ & 273,3 & 316,2 & 312,1 \\
\hline Empresas no-innovadoras & & & $39,18 \%$ \\
\hline
\end{tabular}

Fuente: Encuesta de Actividades de Ciencia y Tecnología

Tabla 8. Gasto en ciencia y tecnología (Valores en millones de dólares corrientes)

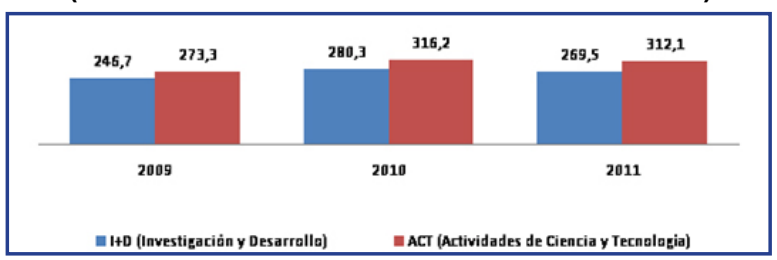

FUENTE: ENCUESTA DE ACTIVIDADES DE INNOVACIÓN

\section{Conclusiones}

La revisión efectuada, evidencia que el Internet es una de las fuentes de información para innovación más frecuentemente usada por los empresarios en Ecuador, gracias a su facilidad y rapidez de acceso a gran cantidad de información que se puede obtener a través de ella(Batraga, 2014).

La tecnología y el Internet se utilizan como fuentes de innovación para las empresas de hoy. Una herramienta importante del Internet son las redes sociales, que han generado incluso la necesidad de su seguimiento permanente, con una persona encargada exclusivamente para dicha tarea, a la que se le ha dado el nombre de community manager.

Una persona encargada de innovar investiga las opiniones que tienen sus clientes por medio de estas redes. De esta manera, también se logra comparar con otros productos similares, y mejorar la calidad de dicho producto(Parga-Dans, 2013).

Mientras el Internet y la tecnología avanzan de la mano, las empresas se apegan a ellas para no retrasarse. Mediante las aplicaciones para teléfonos móviles, las personas encuentran más comentarios de personas y facilitan la comunicación entre los consumidores y las empresas, están así más enteradas de la actualidad, de las opiniones de las personas en base a sus productos y tomando estas opiniones como fuentes para mejorar o innovar(Valls).

El internet posee muchas bondades para las personas encargadas de su seguimiento pero se debe conocer las herramientas disponibles para utilizarlas y así obtener provecho en lo que respecta a tendencias en innovación (Smith, 2014).

\section{Referencias Bibliográficas}

Alvarez, R. Innovación, Investigación y desarrollo. Cepal. (2011).

Batraga, A. Identification of innovation ideas in it is development process. Sisteminai Tyrimai. (2014).

Corma, P. Fuentes de Innovación: Los "cambios en el mercado" . (16 de Septiembre de 2013).

Dirección de Investigaciones y proyectos académicos-DIPA. Plan Estratégico de Investigación Desarrollo Tecnológico E Innovación .Guayaquil: Universidad de Guayaquil. (2012).

Gomez, C. M. Analysis of the relationship between managing and indicators of innovate performance. 
Imperial College Press. (2011).

Heinz, M. Innovation excellence. Obtenido de 10 proven sources of inspiration: http://www.innovationexcellence.com/blog/2011/03/28/10-proven-sources-of-inspiration/ (28 de Marzo de 2011).

Herrera, B. Mediación universitaria en redes locales de innovación. Dialnet. (2013).

INEC. Encuesta de Actividades de Ciencia y Tecnología de la Investigación. Estructura de Indicadores ACTI. (21 de Noviembre de 2013).

Parga-Dans, E. La innovación organizativa y de gestión como motor de dinamización empresarial. Journal of technology managment \& innovation. (2013).

Rivero, F. Fuentes de innovación. MK Marketing + Ventas. (2010).

Salazar, R. Reinventarse para avanzar. Innovando en el área comercial. Navarra, España: IESE Business School. (2013).

Secretaria de Educación Superior, Ciencia y tecnología. Principales indicadores de actividades de ciencia, tecnología e innovación ACTI.INEC. (2009-2011)

Smith, R. Innovation for Innovators. Research-Technology Management. (2014).

Valls, J. (s.f.). The effect of research \& development. Barcelona, España: University of Barcelona. 